Textures and Microstructures, Vol. 33, pp. 173-185 Reprints available directly from the publisher Photocopying permitted by license only
(C) 1999 OPA (Overseas Publishers Association) N.V. Published by license under the Gordon and Breach Science Publishers imprint. Printed in Malaysia.

\title{
BRAGG-EDGE TRANSMISSION AS AN ADDITIONAL TOOL FOR STRAIN MEASUREMENTS
}

\author{
HANS GEORG PRIESMEYER ${ }^{a, *}$, MEINHARD STALDER ${ }^{a}$, \\ SVEN VOGEL ${ }^{a}, K^{a}$ MEGGERS ${ }^{b}$, RALF $^{\text {BLESS }}{ }^{c}$ \\ and WALTER TRELA ${ }^{d}$ \\ ${ }^{a}$ Labor für Angewandte Neutronenphysik/Institut für Experimentelle und \\ Angewandte Physik der Christian-Albrechts-Universität zu Kiel, \\ Olshausenstr. 40, D-24118 Kiel, Germany; ' Siemens AG, SU, \\ P0801760, D-81617 Munich, Germany; ' Ericsson GmbH, \\ Fritz-Vomfelde-Str.26, D 40547 Düsseldorf, Germany; ${ }^{\mathrm{d}}$ P23, \\ Los Alamos National Laboratory, Los Alamos, NM 87545, USA
}

Solid state information from coherent neutron scattering can be extracted from transmission experiments on polycrystalline materials. The transmission technique has been applied to dynamic structural and phase change investigations and will be developed to cover the fields of strain mapping and structural tomography.

Keywords: Neutron diffraction; Transmission; Transient phase transformations; Dynamic phenomena

\section{INTRODUCTION}

A white thermal neutron spectrum, when transmitted through polycrystalline material, is considerably distorted by Bragg edges, which correspond to a scattering angle $2 \theta=180^{\circ}$. Since all diffracted neutrons are scattered out of the direct beam, transmission edges build up rapidly. High peak-intensity neutron bursts can be detected using the currentmode technique, opening the possibility to do both transient and

\footnotetext{
* Corresponding author.
} 
stroboscopic measurements. This new method is relevant to different research areas, like transient phase transformations, melting and solidification, dynamic stress influence at impact or materials under extreme external conditions. An advantage compared to ordinary diffraction is the easier setup of the experiment due to the fact, that the sample can be placed anywhere between the source and the detector. The Bragg-edge method may become a unique tool for the investigation of structural phase-transitions, crystal-structure tomography and strain mapping. Recent developments will also have impact on the determination of the stress-free reference lattice plane distances.

\section{NEUTRON TRANSMISSION DIFFRACTION}

Low-energy coherent neutron scattering from polycrystalline materials can be performed with highest resolution in neutron time-of-flight transmission geometry which corresponds to backscattering, with the only difference that the detectors are placed in the forward direction (Fig. 1). Instead of Bragg peaks so-called Bragg edges are observed. Bragg edges represent the sudden discontinuity in the scattering cross section, whenever certain lattice planes are excluded from scattering since the Bragg condition $\lambda_{h k l}=2 \cdot d_{h k l}\left(\right.$ for $\theta=90^{\circ}$ ) is no more fulfilled. At a powerful pulsed neutron source like LANSCE in Los Alamos, information about the crystal structural properties can be attained from every neutron pulse. Since each pulse contains the full thermalized neutron spectrum, a large number of Bragg reflections can be observed simultaneously.

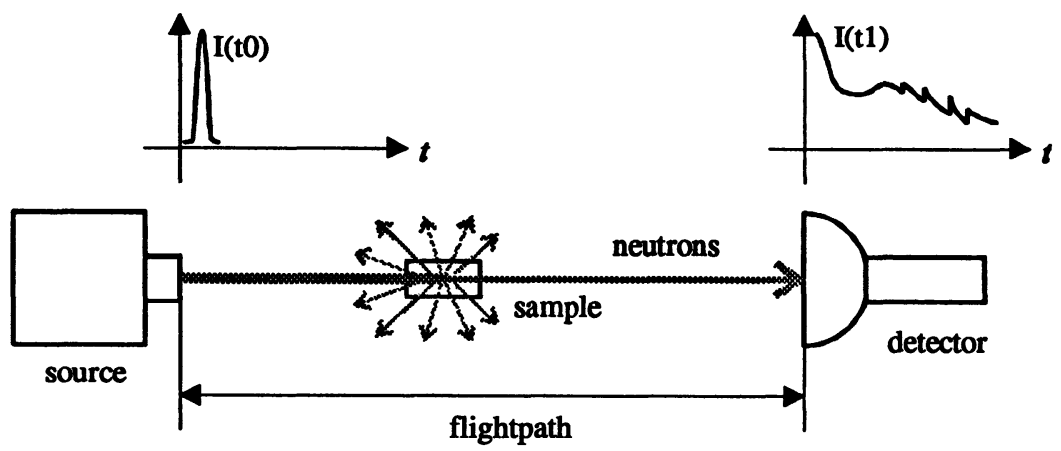

FIGURE 1 Schematic of the transmission setup (from Bless, 1997). 
Real-time investigations of changes in the material properties which influence the position, width and height of the Bragg edges (like phase changes, anisotropic strain development or thermal expansion) can be observed with a time resolution between $100 \mu$ s and several ms. The measured heights must be normalized with respect either to the initial height (for phases with decreasing volume fractions) or to the final height (for phases with increasing volume fractions). One may also "calibrate" the measurements by measuring phase volume fractions in specimens before and after heat treatment using conventional neutron powder diffraction. First experiences with this new method have already been reported (Priesmeyer et al., 1989; Bowman et al., 1991; Priesmeyer, 1992), but further progress has been made concerning single-shot resolution. A new transient recorder capable of registering successive singleshots over minutes has been designed and used to investigate thermal expansion and phase changes in steel, while at the same time a temperature measurement was made by evaluating the Doppler-broadening of the $4.9 \mathrm{eV}$ neutron resonance in a gold foil attached to the steel specimen.

The spectral intensity ratio between "sample-in-beam" and "open beam" is called transmission and described by

$$
T=\exp \left\{-N \cdot \sigma_{\text {coh.-el. }}-n \cdot x \cdot\left(\sigma_{\text {inc. }}+\sigma_{\text {abs. }}\right)\right\}
$$

where $N$ is the number of unit cells $/ \mathrm{cm}^{3}$ and $n$ is the number of atoms $/ \mathrm{cm}^{3}, x$ the thickness of the specimen, $\sigma_{\text {coh.el. }}$ the coherent-elastic cross section, $\sigma_{\text {inc. }}$ the incoherent cross section and $\sigma_{\text {abs. }}$ the absorption cross section.

The coherent-elastic scattering cross section is given by

$$
\sigma_{\tau}(\lambda)=\frac{\lambda^{2} \cdot z_{\tau}}{2 \cdot V_{\text {cell }}} \cdot|F(\tau)|^{2} \cdot \mathrm{e}^{-2 W} \cdot d_{\tau} \quad \text { and } \quad \sigma_{\text {coh.-el. }}(\lambda)=\sum_{\tau \text { for } 2 d_{\tau}>\lambda} \sigma_{\tau}(\lambda)
$$

where the unit-cell structure factor

$$
F(\tau)=\sum_{i} \bar{b}_{i} \cdot \exp (2 \pi i \tau \cdot d) \cdot \exp \left(-2 W_{i}\right)
$$


comprises the nuclear properties of the scatterer, crystal structure selection rules and multiplicities as well as thermal effects, described by the Debye-Waller factor.

\section{THE PRECISION LIMITS OF BRAGG-EDGE PARAMETERS}

In the field of residual stress assessment using neutron diffraction, lattice spacings must be determined with a error of less than $10^{-4}$. Residual stress states exist in components even if they are not subjected to external load. They develop in a very complicated, highly coupled thermal/metallurgical/mechanical process during welding, casting or forging or as a result of other manufacturing processes. Recent research (Priesmeyer et al., 1994) on the development of residual stresses includes their calculation, for which certain material constants are important to know. These include the time development of the buildup of incompatible phases as well as the interrelation between phase transformation kinetics and internal stress, so-called transformation strains.

The relative reflex intensities yield information on phase volume fractions (Meggers et al., 1994a,b). In transmission experiments the high precision attained in ordinary backscattering geometry concerning profile parameters of Bragg edges is preserved. Two-dimensional macroand microstrain can be accurately measured. Figure 2 shows a section of a typical current-mode single-shot transmission spectrum for $\alpha$-iron (bcc) of $2.5 \mathrm{~cm}$ thickness, collected at $10 \mathrm{~m}$ detector position within $7 \mathrm{~ms}$. In order to improve the statistical accuracy, multiple single-shots can be accumulated.

Edge height, slope and position are extracted from the transmission spectra. The shape of Bragg edges is generally a convolution of the contributions of the natural and mosaic spread of lattice spacings, the resolution function and strain gradient influences. An evaluation procedure similar to conventional Rietveld refinement is being developed. As a first attempt to use existing software, transmission spectra were differentiated. The resulting peaks were fitted as single peaks, while differentiation tends to increase noise and distort the continous background (Fig. 3).

The parameters describing the resolution function are also fitted. Accuracies achieved for peak positions where about $4 \cdot 10^{-4}$ for a measuring time of $250 \mathrm{~s}$, but can further be increased. 
current intensity (rel.units)

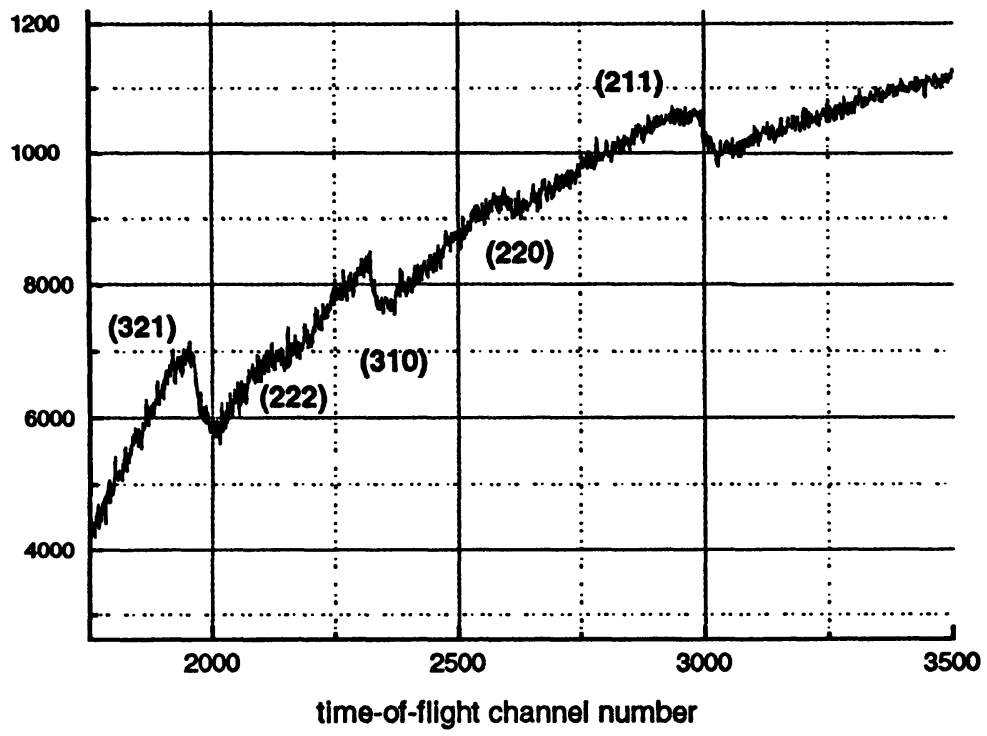

FIGURE 2 Partial single-shot spectrum at $10 \mathrm{~m}$ flight-path.
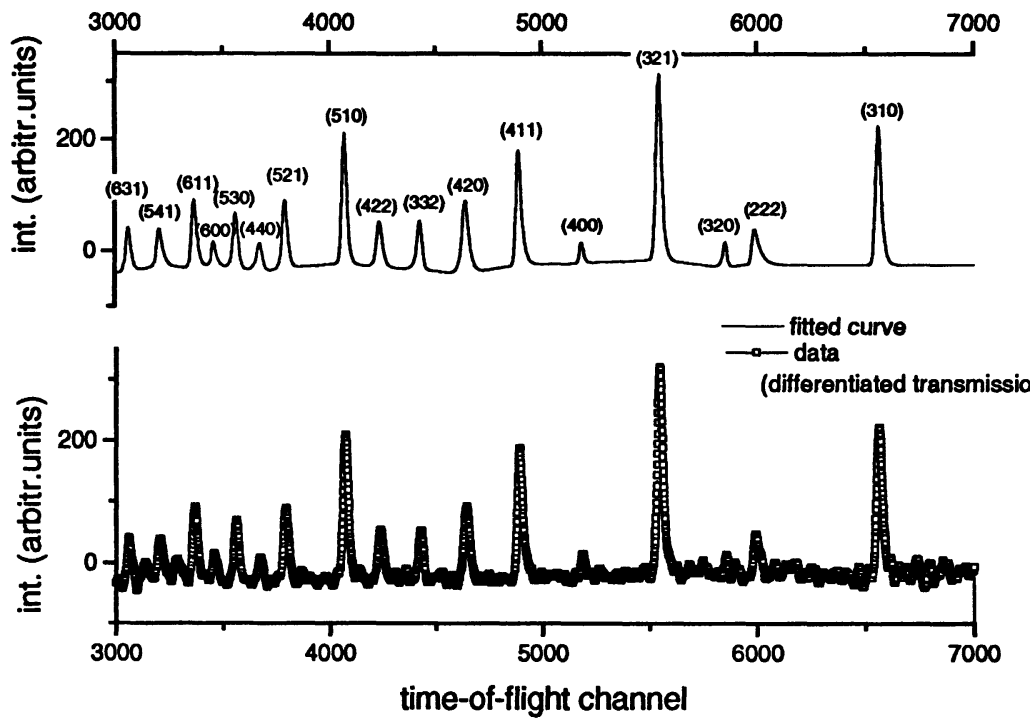

FIGURE 3 Differentiated time-of-flight transmission spectrum (bottom) and fit (top) of bcc-iron (from Vogel, 1996). 


\section{TEMPERATURE MEASUREMENT BY}

\section{DOPPLER-BROADENING OF NUCLEAR RESONANCES}

Many of the possible applications of single pulse transmission (like solid state phase changes, development of transformation stresses, thermal stresses during cooling after welding) require the knowledge of the temperature of the specimen. It is known that Doppler-broadening of low-lying neutron resonances can be exploited to determine temperatures. A series of experiments has been made using ferritic steel together with a $17 \mu \mathrm{m}$ thick gold foil. Figure 4 shows a current-mode detection spectrum containing both edges and a resonance.

Both resonance Doppler-broadening and Bragg-edge shift are functions of the temperature of a specimen. While the position of the Bragg edges is influenced by the combined effects of thermal lattice expansion and strain a resonance is only subjected to thermal Doppler-broadening. In technological applications it may not be possible to mix dopant and alloy without changing the metallurgical properties of the latter. Details of spectral differences due to temperature are shown in Figs. 5 and 6.

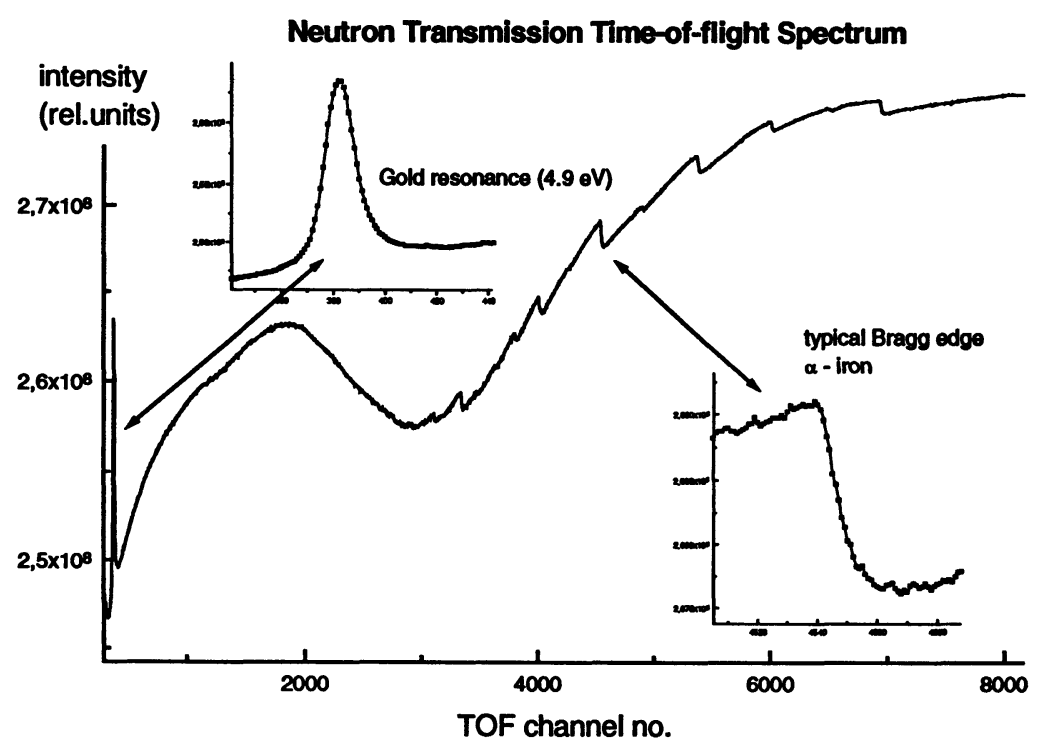

FIGURE 4 Simultaneous current-mode recording of the $4.9 \mathrm{eV}$ gold resonance and several edges of bcc-iron. 


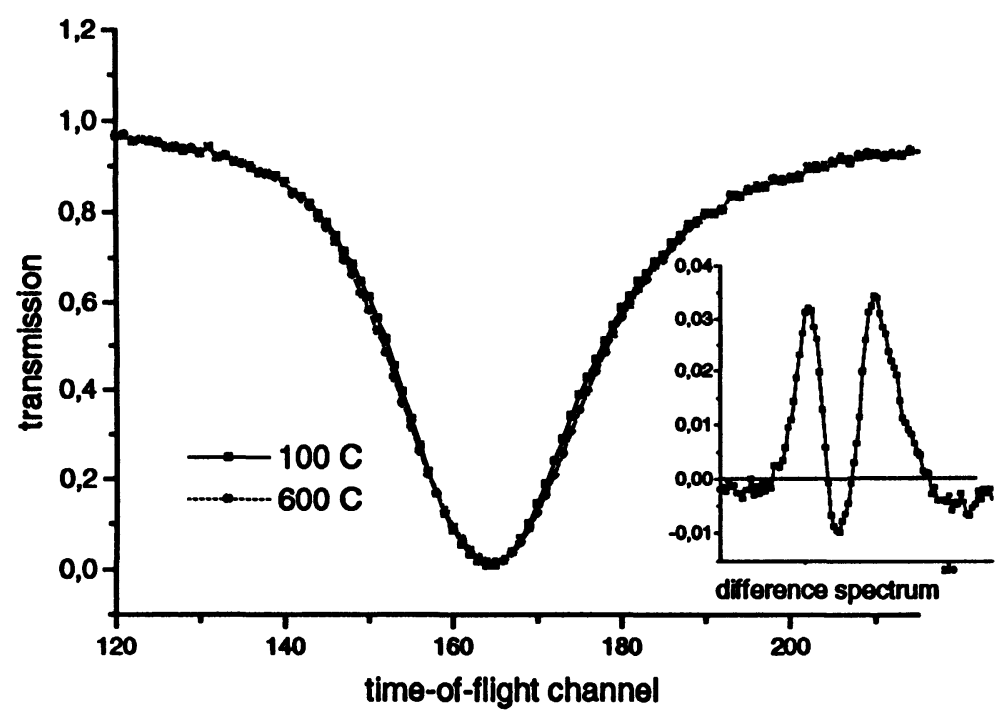

FIGURE 5 Resonance broadening between $100^{\circ} \mathrm{C}$ and $600^{\circ} \mathrm{C}$.

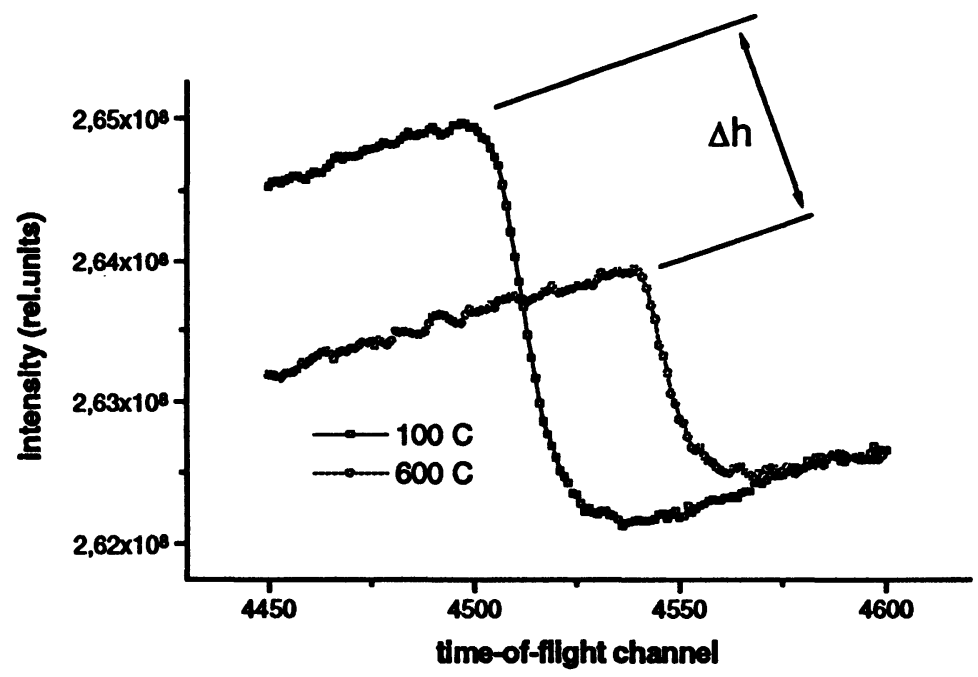

FIGURE 6 (321)-reflection in bcc-iron, shifted in time-of-flight by thermal expansion and reduced in height by $\Delta h$ according to the Debye-Waller factor. 


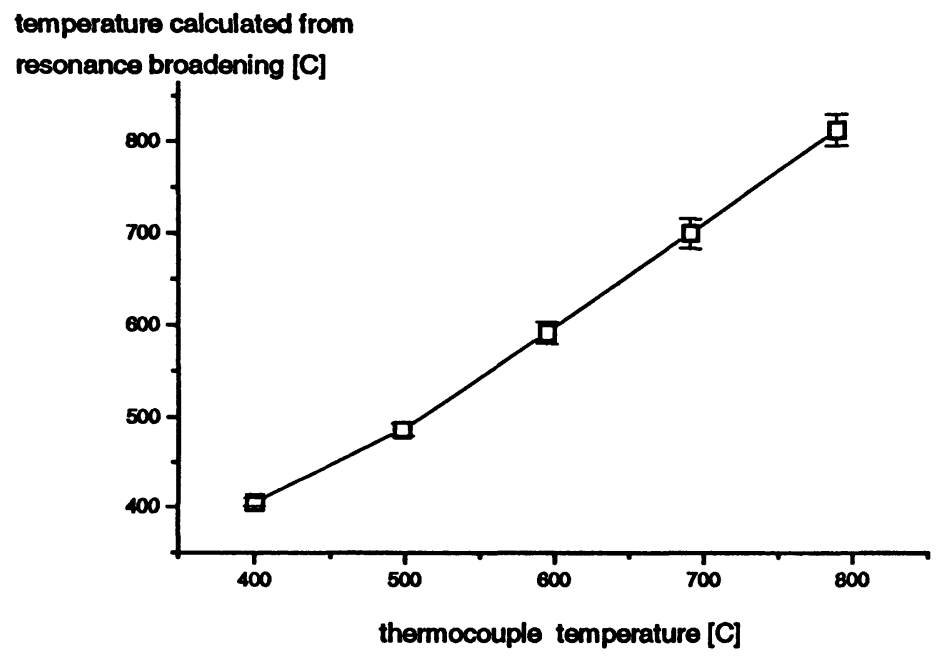

FIGURE 7 Comparison between temperatures derived from thermocouple and resonance broadening measurements (from Stalder, 1996).

Bragg edges may vary in height for two reasons: the Debye-Waller factor will decrease the diffracted intensity with increasing temperature and diffusion-controlled phase changes may reduce the volume fraction of a certain phase. In steel up to $600^{\circ} \mathrm{C}$ intensity reduction can be fully attributed to the Debye-Waller factor. The principle feasibility of the method has been demonstrated by a series of measurements from room temperature to $1000^{\circ} \mathrm{C}$. A better knowledge of the spectrometer resolution function and of the open beam intensity will be needed to arrive at shorter times. In order to derive temperature values from resonance broadening, the method described by Mayers et al. (1989) was used. Resonance shape fitting will be used later.

\section{PIEZOELECTRIC STRAIN GAUGE FOR STATIC AND DYNAMIC LOAD}

A stress rig has been developed suitable for transmission experiments. In order to adopt it to a pulsed neutron source, a special device was designed on the basis of an annular piezoelectric actuator (Bless, 1997, patent pend.). 


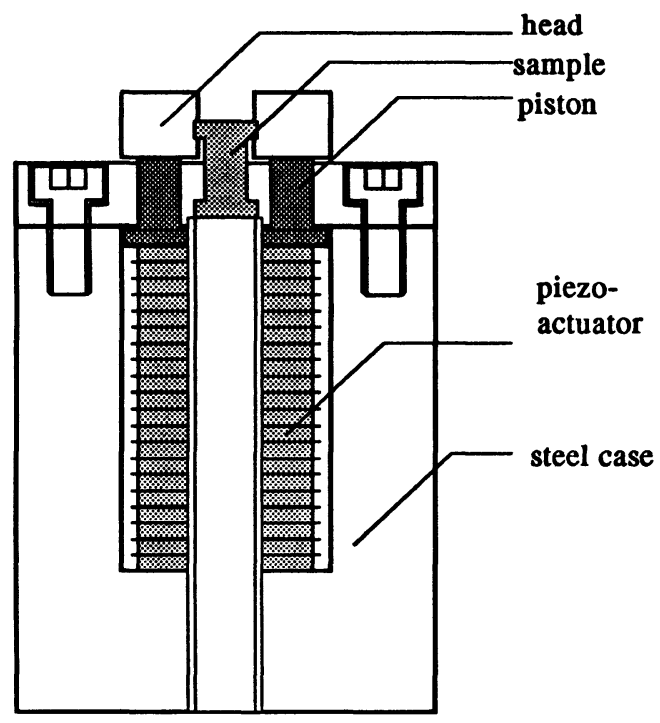

FIGURE 8 Schematic of the piezoelectric uniaxial stress rig (from Bless, 1997).

The outlines are shown in Fig. 8.

The rig can be triggered by the neutron source and the load be applied at certain time intervals; different strain rates can be used to investigate strain-rate dependent effects. The dynamic features were tested using the setup shown in Fig. 9.

For a mechanically prestrained steel specimen, the neutron diffraction result vs. strain gauge comparison is given in Fig. 10.

\section{BRAGG-EDGE TRANSMISSION SPECTROSCOPY TO DETERMINE THE STRESS-FREE REFERENCE LATTICE SPACING $d_{0}$}

Under the assumption of a two-dimensional stress state (e.g. thin specimens), Bragg-edge transmission measurements may offer an opportunity for in situ measurements of $d_{0}$. Strain in the direction determined by the angles $\phi$ and $\psi$ can be described by the following well known 


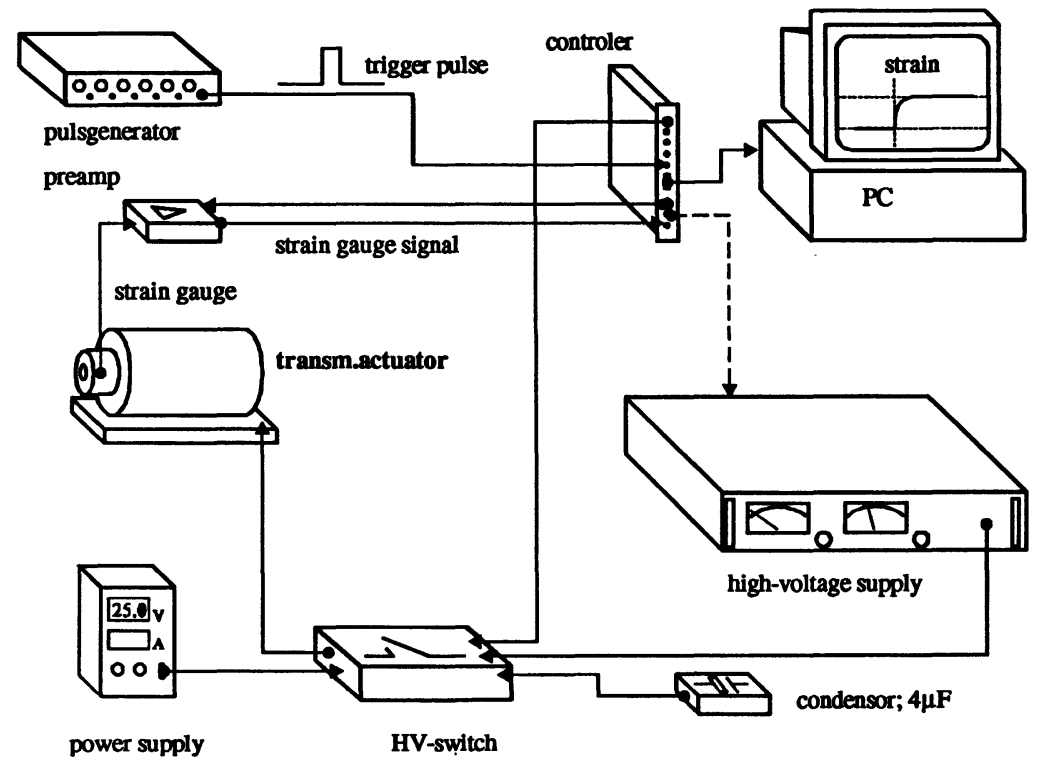

FIGURE 9 Setup to characterize the dynamic features of the transmission stress rig (from Bless, 1997).

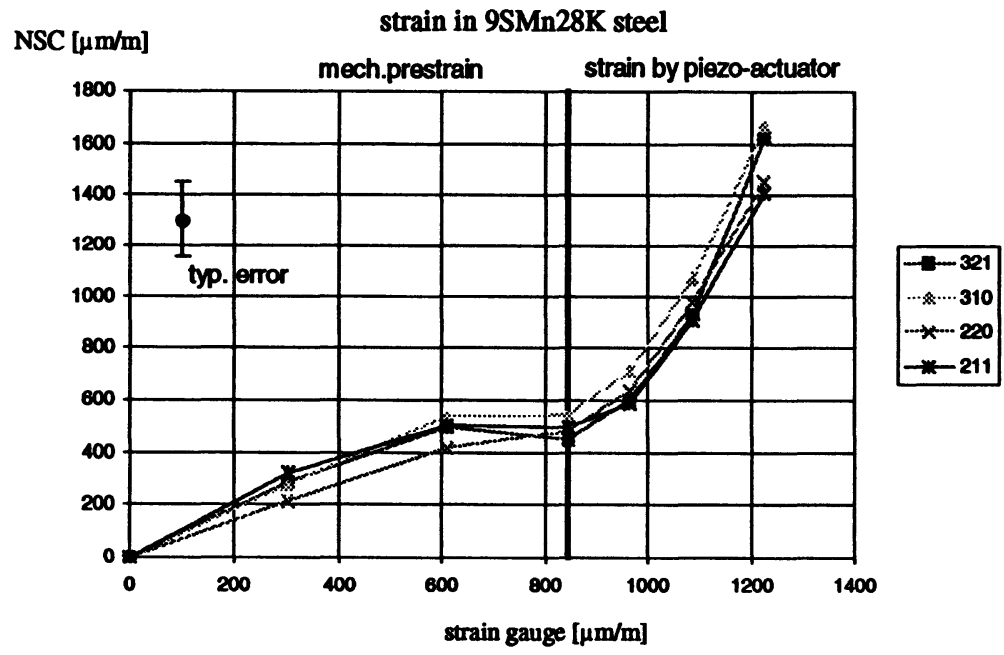

FIGURE 10 Strain in steel specimen: mechanical prestrain (left side), piezoelectrically applied strain (right side) (Bless, 1997). 
expression:

$$
\begin{aligned}
\frac{d_{\phi, \psi}-d_{0}}{d_{0}}= & \frac{1+\nu}{E} \cdot\left\{\sigma_{x x} \cdot \cos ^{2}(\phi)+\sigma_{x y} \cdot \sin (2 \phi)+\sigma_{y y} \cdot \sin ^{2}(\phi)-\sigma_{z z}\right\} \\
& \cdot \sin ^{2}(\psi)+\frac{1+\nu}{E} \cdot \sigma_{z z}-\frac{\nu}{E} \cdot\left\{\sigma_{x x}+\sigma_{y y}+\sigma_{z z}\right\} \\
& +\frac{1+\nu}{E} \cdot\left\{\sigma_{x z} \cdot \cos (\phi)+\sigma_{y z} \cdot \sin (\phi)\right\} \cdot \sin (2 \psi)
\end{aligned}
$$

Assuming biaxial stress $\left(\sigma_{z z}=0\right)$ and taking $\phi=0$, the equation further simplifies to:

$$
\frac{d_{\phi, \psi}-d_{0}}{d_{0}}=\frac{1+\nu}{E} \cdot \sigma_{x x} \cdot \sin ^{2}(\psi)-\frac{\nu}{E} \cdot\left\{\sigma_{x x}+\sigma_{y y}\right\}
$$

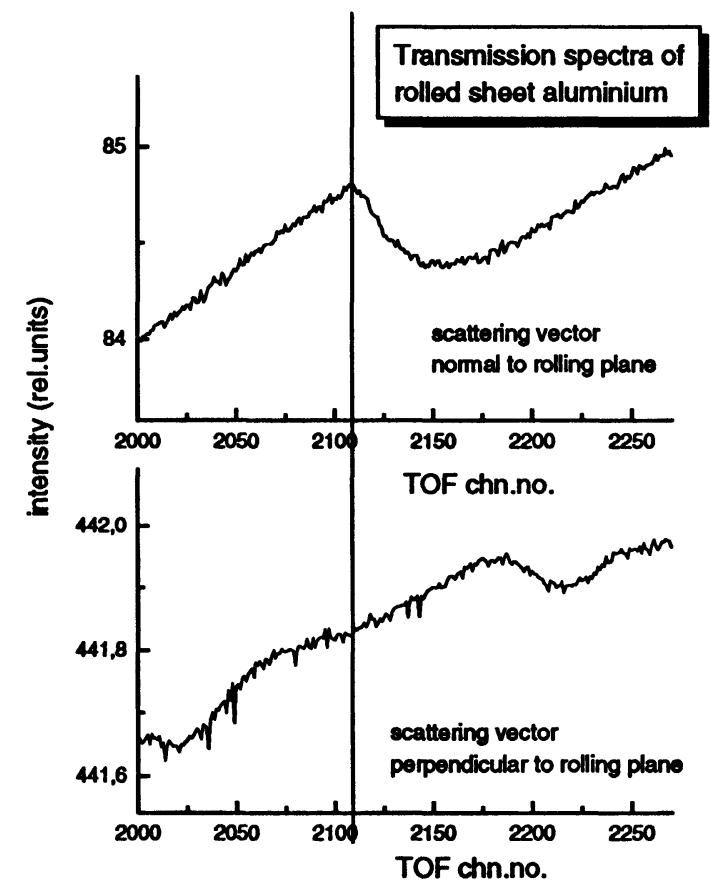

FIGURE 11 Neutron transmission near the (311)-edge in aluminium for two orthogonal positions of the specimen. 
The angle $\psi=\psi^{*}$ for which $d_{\psi^{*}}=d_{0}$ may be calculated from the following relation:

$$
\sin ^{2}\left(\psi^{*}\right)=\frac{\nu / E}{(1+\nu) / E} \cdot\left\{1+\frac{\sigma_{y y}}{\sigma_{x x}}\right\}=\frac{\nu / E}{(1+\nu) / E} \cdot\left(1+\frac{m_{y}}{m_{x}}\right)
$$

where $m_{x}$ and $m_{y}$ are the slopes of the $d\left(\sin ^{2}(\psi)\right)$ curves, measured for two orthogonal directions $\phi=0^{\circ}$ and $\phi=90^{\circ}$. A plot of measured $d$ spacings vs. $\sin ^{2}(\psi)$ for any two $\phi_{A}$ and $\phi_{A}+90^{\circ}$ will lead to the relevant slopes and the angle $\psi=\psi^{*}$.

\section{BRAGG-EDGE TRANSMISSION AND TEXTURE}

A recent measurement of the transmission of rolled sheet aluminium at LANSCE has shown how texture can considerably change the shape of a neutron transmission spectrum (Fig. 11). Evaluation of these findings is still in progress. In general, it should be possible to derive information about the textured state. Since for in-depth strain profiling the neutrons penetrate a certain material layer, consequences for the interpretation of strain data in textured materials may also be anticipated.

\section{Acknowledgements}

This work was financially supported by Deutsche Forschungsgemeinschaft AZ Pr267/3-3 and LANCSE, Los Alamos National Laboratory.

\section{References}

Bless, R. (1997) Ein elektronisch gesteuerte Zugvorrichtung zur Untersuchung elastischer Werkstoffkennwerte mit Hilfe der Neutronen-Transmissions-Diffraktometrie, Exp.-Phys. Diplomarbeit, Christian-Albrechts-Universitat zu Kiel.

Bowman, C.D., Egelstaff, P.A. and Priesmeyer, H.G. (1991) "Experiments using single neutron pulses”, ICANS-XI KEK Report 90-25 March 1991, AMRD Vol. II, 840 ff. M. Misawa, M. Furusaka, H. Ikeda and N. Watanabe, Eds.

Fowler, P.H. and Taylor, A.D. (1993) LA 11393-C Conference (UC 414), pp. 46-80.

Mayers, J., Baciocco, G. and Hannon, A.C. (1989) "Temperature measurement by neutron resonance radiography”, Nuclear Instr. Methods in Physics Research A 275, 453-459.

Meggers, K., Priesmeyer, H.G., Trela, W., Bowman, C.D. and Dahms, M. (1994a) “Realtime neutron transmission investigation of the austenite-bainite transformation in gray iron", Nuclear Instr. Methods in Physics Research B 88, 423-429. 
Meggers, K., Priesmeyer, H.G., Trela, W. and Dahms, M. (1994b) "Investigation of the austenite-bainite transformation in gray iron using real-time neutron transmission", Materials Science and Engineering A 188, 301-304.

Priesmeyer, H.G., Bowman, C.D., Yuan, V.W., Seestrom, S.J., Wender, S.A., Richardson, O.A., Wasson,, O.A., Xzu, X. and Egelstaff, P.A. (1989) "Fast transient neutron diffraction at LANSCE”, Los Alamos Memo 1989 (unpublished).

Priesmeyer, H.G. (1992) "Transmission Bragg-edge measurements", Measurement of Residual and Applied Stress Using Neutron Diffraction, Proceedings NATO ARW Oxford, M.T. Hutchings and A.D. Krawitz Eds. (Kluwer, Dordrecht, 1992) pp. 389394.

Priesmeyer, H.G., Larsen, J. and Meggers, K. (1994) "Neutron diffraction for nondestructive strain/stress measurements in industrial devices", Journal of Neutron Research, 2(1) 31-52.

Priesmeyer, H.G., Stalder, M., Vogel, S., Meggers, K. and Trela, W. (1997) "Progress in single shot neutron transmission diffraction", 5th International Conference on Application of Nuclear Techniques, "Neutrons in Research and Industry", Crete 1996, SPIE, 2867, $164-167$.

Stalder, M. (1996) "Neutronentransmissionsuntersuchungen an Stahl und Temperaturbestimmung mithilfe der resonance-doping-method", Exp.-Phys. Diplomarbeit, Christian-Albrechts-Universität zu Kiel.

Vogel, S. (1996) "Neutronendiffraktometrie in Transmissionsgeometrie mit einem Transientenrekorder”, Exp.-Phys. Diplomarbeit, Christian-Albrechts-Universität zu Kiel. 